\title{
Selective attention versus selection for action: Negative priming is not the result of distractors being unattended
}

\author{
STEVE JOORDENS and ITANNI BETANCOURT \\ University of Toronto Scarborough, Toronto, Ontario, Canada \\ and \\ THOMAS M. SPALEK \\ Simon Fraser University, Burmaby, British Columbia, Canada
}

\begin{abstract}
Using a novel referent size-selection task, MacDonald, Joordens, and Seergobin (1999; MacDonald \& Joordens, 2000) found that negative priming persisted even when participants were encouraged to attend to distractors before selectively responding to targets. This finding suggested that negative priming is not caused by processes that operate on stimuli that are to be ignored in the traditional selective attention sense. Mackintosh, Mathews, and Holden's (2002) attempt to replicate the MacDonald et al. study resulted in the discovery of possible artifacts in the referent size-selection task, thereby making the implications with respect to the role of attention less clear. In the present study, we describe a different method for directing attention to distractors in a negative priming context, one that does not suffer from the same potential artifacts as the referent size-selection task. Our results are consistent with those found by MacDonald et al., in that negative priming persisted even when participants were explicitly encouraged to attend to distractors. Implications are discussed in the context of the related concepts of selective attention (e.g., Broadbent, 1965) versus selection for action (e.g., Allport, 1987).
\end{abstract}

The classic literature on selective attention (e.g., Broadbent, 1965; Treisman, 1964) defined the area as the study of the cognitive mechanisms used to deploy attention toward certain stimuli and away from others. According to this early work, the purpose of these cognitive mechanisms was to segregate (i.e., select) which stimuli would gain access to awareness via attentional processing and which would not. For example, with his now-famous cocktailparty phenomenon, Cherry (1953; Cherry \& Taylor, 1954) highlighted the ease with which an individual can segregate two auditory streams. The result of this segregation is that the individual is aware of the semantic content of the attended message but not of the unattended message. Treisman, Squire, and Green (1974) make a similar point while discussing the potential role of attention shifts that may occur in the context of dichotic listening tasks. Specifically, they state that the identification of an unattended word does not "inevitably bring with it a shift of attention, conscious awareness, and storage in memory, but . . may do so" (pp. 641-642). The implication is that, when atten-

This research was supported by an NSERC Discovery Grant and a Premier's Research Excellence Award, both awarded to the first author. We thank Trammel W. Neill and an anonymous reviewer for their extremely constructive reviews of earlier versions of this manuscript. Correspondence should be addressed to S. Joordens, Department of Life Sciences, University of Toronto Scarborough, 1265 Military Trail, Toronto, ON, M1C 1A4 Canada (e-mail: joordens@psych.utoronto.ca). tion shifts to unattended items, the result is awareness of those items.

Others have discussed a related but distinct concept termed selection for action (e.g., Allport, 1987; Treisman, 1996; van der Heijden, 1996). The selection-for-action notion is subtly different from the traditional selective-attention concept. Whereas the emphasis in the selective-attention concept is on a mechanism that segregates stimuli into categories of attended versus unattended, the selectionfor-action concept instead emphasizes a mechanism that segregates stimuli into categories of to-be-acted-on stimuli versus not-to-be-acted-on stimuli. Given this, the selectionfor-action concept could be described as noncommittal with respect to what role, if any, attention or awareness may play in the selection process. Thus, within the selection-foraction notion, it is reasonable to think of a stimulus as being attended but not responded to, or perhaps even unattended but responded to. This contrasts markedly with the Treisman et al. (1974) quote in the previous paragraph in which response processes were not discussed at all, and the focus was clearly on the cognitive processes that may differ between items that were or were not attended.

The differences between these two perspectives has, in our opinion, lead to some confusion within the context of the negative priming phenomenon. Tipper and colleagues (e.g., Tipper, Howard, \& Houghton, 1999; Tipper, Weaver, \& Houghton, 1994) have been the major proponents for the selection-for-action perspective of negative priming. However, while drawing implications primarily within 
the selection-for-action framework, a relevance to more classical notions of selective attention is often implied. For example, the title of Tipper et al.'s (1994) article is "Behavioural Goals Determine Inhibitory Mechanisms of Selective Attention." In fact, Tipper has also described negative priming as occurring when one must currently respond to an item that was unattended on a previous display. Are such claims accurate? Does negative priming in any way depend on distractors being unattended?

Unfortunately, given the typical procedures used to produce negative priming, an easy answer to this question is not readily apparent. That is, most negative priming studies use procedures in which a target and a distractor are presented simultaneously and participants are instructed to selectively attend and respond to the target while ignoring and not responding to the distractor. This procedure begs for answers to several questions. Do the participants ignore the distractor? Is the distractor unattended? If so, is this unattended status critical for observing the negative priming effect? All we know for certain is that participants are generally quite able to respond to the target while not responding to the distractor. Aside from that we have very little knowledge about how the distractor may have been processed and what role attention or inattention might play with respect to determining negative priming.

As mentioned earlier, the selection-for-action notion allows for the possibility that stimuli can be attended, perhaps to the extent that participants are explicitly aware of their identity, but could ultimately be selected against in an action sense at later stages of processing. If negative priming were observed in such a context, it would clearly indicate that the action status of the two stimuli (one to be acted on and one not) gave rise to negative priming, and not the attentional status (one being actively attended and the other not). The typical procedures confound the attentional versus response demands placed on participants, given that distractors are to be both "ignored" and "not responded to." To understand what role attention may play with respect to negative priming, a variant of the procedure is required in which participants attend to, but do not respond to, distractors.

One such variant of the typical negative priming procedure has been described in the literature. MacDonald, Joordens, and Seergobin (1999; see also MacDonald \& Joordens, 2000) have published work suggesting that negative priming not only persists when distractors are attended but that, in fact, the negative priming effect actually becomes much larger in such contexts. However, findings by Mackintosh, Mathews, and Holden (2002) have suggested that artifacts may have existed in the procedural variation described by MacDonald et al. (1999), thus questioning the validity of MacDonald et al.'s conclusions.

Our goal in the present work was to revisit this issue using a different procedural variant. To provide a context for our work, we first briefly describe the typical negative priming procedures and then describe the variant employed by MacDonald et al. (1999). We then discuss the attempt by Mackintosh et al. (2002) to replicate the MacDonald et al. study, while emphasizing what those find- ings do and do not say about the potential role of attention with respect to negative priming. Finally, we present two experiments that further support the notion that negative priming does occur when distractors are attended.

\section{Typical Procedures}

A typical negative priming procedure requires selective responding to a target item presented among one or more distractors on a first trial (i.e., the prime trial). The task is then normally repeated on a second trial (i.e., the probe trial). The critical element is the relation between the targets and the distractors on the consecutive prime and probe trials. In the control condition, no relation exists between the targets and the distractors on the two consecutive trials. Hence, different, unrelated distractors and targets occur on the prime and probe trials. This condition serves as the baseline to which the latency and error rates of the experimental conditions are compared. The ignored repetition condition is the critical experimental condition. In this condition, the item that served as the distractor on the prime trial is the target on the probe trial. Lastly, some negative priming experiments also include an attended repetition condition in which the target on the probe trial is a repetition of the target that had been presented on the prime trial.

The main empirical finding is that responses are slower and more error prone on ignored repetition trials, relative to control trials. This finding was originally noted by Dalrymple-Alford and Budayr (1966), followed up by Neill (1977), and ultimately termed negative priming by Tipper (1985). In addition, participants tend to respond faster and are less error prone on attended repetition relative to control trials (e.g., Milliken \& Joordens, 1996). Thus, when the current target was ignored and not responded to on the previous trials, responses are relatively inefficient. But when the current target was attended and responded to on the previous trial, responses are relatively efficient. The question remains: Is this qualitative difference in response patterns due to differential attentional processing or to something that occurs farther downstream in processing?

\section{The Referent Size-Selection Task}

In an attempt to clarify some of the described ambiguity regarding the relation between negative priming and attention, MacDonald et al. (1999) created a situation in which both the target and the distractor were required to be attended on both the prime trials and the probe trials. This was accomplished using a novel referent sizeselection task in which participants were instructed to name the larger animal in a presented animal pair (e.g., DOG-FLEA). This task ensured that both targets and distractors were attended, were processed deeply (i.e., compared on a semantic dimension), and reached phenomenal awareness on all trials. However, only the targets were responded to (i.e., named aloud). Thus, the distractors were attended, but then no response was made to these items.

Despite the fact that the distractors were attended, negative priming was observed on this new task. In fact, a direct comparison of the typical task (i.e., name the red item in the pair) with the referent size-selection task (i.e., 
name the larger item in the pair) revealed that the effect was nearly four times as large in the size-selection context. This finding runs counter to the notion that negative priming is caused by not attending to distractors. In fact, it suggests that attending to distractors somehow enhances the phenomenon.

For present purposes, we wish to emphasize that there are two components to this conclusion. First is the notion that negative priming does not result from a lack of attention to the distractor item, because even attended distractors gave rise to negative priming. Second is the notion that attending to distractors may actually enhance the effect. We see these as two separate issues that are both compromised by concerns regarding the referent sizeselection task that will be described later, and both are relevant to the experiments we present here.

A follow-up study by Mackintosh et al. (2002) has cast a shadow of doubt over the MacDonald et al. (1999) conclusions by suggesting that the referent size-selection task may suffer from an artifact. Specifically, the stimuli used by MacDonald et al. were FLEA, MOUSE, TURTLE, PIG, GOAT, DONKEY, BEAR, and CAMEL. Recall that these stimuli were always presented in pairs, and, in the context of the referent size-selection task, participants are asked to name the "larger" member of the pair. This task creates constraints that prevent some of the stimuli from being in all conditions. Specifically, because FLEA is the smallest animal in the set, it can never serve as a target, given that it can never be the larger of two animals. Conversely, CAMEL is the largest animal and, therefore, can never serve as a distractor. These constraints prevent either of these items from being used on ignored repetition trials where the item must serve as both a distractor in the prime trial (which CAMEL can never be) and a target in the probe trial (which FLEA can never be).

MacDonald et al. (1999) dealt with this dilemma in the following manner. In the control condition, all of the stimuli, other than FLEA and CAMEL, appeared equally often as targets and as distractors. The word FLEA served as a distractor twice as often as the other stimuli because it could never be a target, and CAMEL served as a target twice as often as the other stimuli because it could never be a distractor. On the other hand, in the ignored repetition condition, CAMEL and FLEA never served as distractors on the prime trials or as targets on the probe trials. CAMEL could also never serve as a probe distractor, and FLEA could never serve as a prime target, because of their relative sizes. CAMEL was used as a prime target approximately twice as often as the other stimuli, and FLEA was used as a probe distractor approximately twice as often as the other stimuli. Therefore, the constraints of the referent size-selection task with regard to these endpoint stimuli resulted in inequalities across the conditions.

In their initial attempts to replicate the referent sizeselection findings, Mackintosh et al. (2002) dealt with the endpoint stimuli different from how MacDonald et al. (1999) dealt with the endpoint stimuli. Mackintosh et al. failed to replicate the enhanced negative priming effect. After personal communication with MacDonald, they iso- lated the different treatments with respect to the endpoint stimuli as the most likely explanation for the failure to replicate. Specifically, they noticed that decisions on trials that included endpoint stimuli (i.e., CAMEL or FLEA) were made faster than were decisions on trials in which both stimuli were from the middle of the size continuum. Given this, Mackintosh et al. pointed out that the stimulus assignment used by MacDonald et al. might have inadvertently given rise to the augmented negative priming effect that they observed via the relatively large number of "fast" endpoint stimuli in MacDonald et al.'s control condition. Thus, Mackintosh et al. argued that the negative priming effect was enhanced not because of disproportionately slow responses on ignored repetition trials but, rather, because of disproportionately fast responses on control trials.

In their study, Mackintosh et al. (2002) contrasted response patterns observed when stimuli were assigned in the manner used by MacDonald et al. (1999) with response patterns observed when the endpoint stimuli were better controlled. Their findings showed large negative priming effects when the assignment was performed according to the guidelines provided by MacDonald et al. However, these negative priming effects either disappeared or were substantially reduced when their stimulus assignment was used.

One could debate which stimulus assignment is truly superior, but, in the broader sense, this debate would be fruitless. Irrespective of the details, Mackintosh et al. (2002) have clearly shown that the stimulus assignments used in conjunction with the referent size-selection task matter, and this fact alone makes the implications derived from that task less clear. Given this potential for artifacts when using the referent size-selection task, our goal in the present work was to revisit the issue of the role of attention on negative priming, in the context of procedures that encourage participants to attend to distractors but that do not suffer from artifact issues.

\section{Present Study}

We describe a novel procedure designed to encourage participants to attend to the distractor items in a negative priming context. To anticipate, the results showed that negative priming does occur even when participants are encouraged to attend to distractors. Thus, our findings provide support for the conclusion that negative priming does not result from the critical items being unattended.

\section{EXPERIMENTS 1A AND 1B}

In Experiments 1A and 1B, our objective was to compare the results obtained using the typical negative priming instructions (i.e., the ignore-distractor condition) with those achieved in a variant procedure that encouraged participants to attend to distractors prior to responding to the targets (i.e., the monitor-distractor condition). In both conditions, the primary task involved the presentation of overlapping red and white numbers with participants instructed to categorize the red number as less than or equal to 4 or greater than or equal to 5 (i.e., a low/high 
decision). The target numbers were always red and were numbered 1-8. The distractor items were always white and usually consisted of the numbers 1-8; however, occasionally, the number 9 (Experiment 1A) or the letter $\mathrm{P}$ (Experiment 1B) was presented as a distractor.

The participants in the ignore-distractor condition were not explicitly informed of the occasional presence of the number 9 or letter $\mathrm{P}$ stimulus. The participants in the monitor-distractor condition were told that "white $9 \mathrm{~s}$ " (or "white Ps" in Experiment 1B) would sometimes appear as distractors and that, when they noticed one, they were to strike the space bar rather than make a low/high decision to the red target item. Thus, the participants in the monitor-distractor condition had to monitor the distractors, to ensure that they responded appropriately. The critical question was what effect this monitoring had with respect to distractors that were not number $9 \mathrm{~s}$ or letter Ps (i.e., attended distractors that were not responded to) and that subsequently became probe targets.

Across Experiments $1 \mathrm{~A}$ and $1 \mathrm{~B}$, we also examined whether it mattered whether the participants were asked to monitor for the letter P or the number 9. Given that the primary task required the participants to make low versus high decisions with respect to a 1-to- 8 continuum, it is possible that using a response-relevant distractor (i.e., one that might be associated with a "high" response) might differentially affect our findings. In addition, by examining both conditions, we were able to realize the potential to replicate and slightly generalize any results. The 9 -versus-P contrast was chosen simply because these two stimuli share similar structural properties but different semantic properties.

\section{Method}

Participants. Fifty-one volunteers from an introductory psychology class at the University of Toronto at Scarborough took part in the experiment in exchange for course credit. Twenty-four participants were randomly assigned to Experiment $1 \mathrm{~A}$, and 27 participants were assigned to Experiment 1B. In Experiment 1A, 12 participants were randomly assigned to the ignore-distractor condition, and 12 were randomly assigned to the monitor-distractor condition. In Experiment 1B, 12 participants were randomly assigned to the ignore-distractor condition, and 15 were randomly assigned to the monitor-distractor condition. All participants had normal or corrected-to-normal vision, and all were sufficiently proficient in English to complete the task without difficulty.

Procedure. On 16 of the 18 prime and probe displays presented within each block, two overlapping numbers that ranged from 1 to 8 appeared in the center of the screen, with one number presented slightly more upward and to the left, and the other presented slightly more downward and to the right. One number was red, and the other was white. On the remaining 2 trials, the setup was identical except that the prime distractor was replaced either with the number 9 (Experiment 1A) or with the letter P (Experiment 1B).

In the ignore-distractor condition, the participants were instructed to press the " $Z$ " key on the keyboard if the red number was $1,2,3$, or 4 and to press the " " key if it was $5,6,7$, or 8 . The task was the same for the participants in the monitor-distractor condition except that they were further instructed to monitor the white numbers (i.e., the distractors) in search of randomly appearing number 9s (Experiment 1A) or Ps (Experiment 1B). In the case that a number 9 (or P) appeared as a distractor, they were to press the space bar rather than perform the low/high discrimination.
Note that the 9s (Experiment 1A) and Ps (Experiment 1B) also appeared during the ignore-distractor condition, but the participants were not informed of them. Given that the participants were only responding to the red stimuli in the ignore-distractor condition, the presence of the $9 \mathrm{~s}$ or Ps need not affect their ability to make their discriminations. Thus, the stimulus presentation was identical across the conditions; only the instructions to monitor were added to the monitor-distractor condition.

Each trial consisted of both a prime and a probe display, and it proceeded as follows. The overlapping red and white numbers were presented and remained visible until the participant responded. If the participant made an error, a biphasic error tone was sounded for $500 \mathrm{msec}$ and the word INCORRECT appeared in the center of the screen. Following a correct response, or immediately after the error signal, the next stimulus display was automatically initiated.

The participants received 16 experimental blocks, each consisting of 18 trials. Of these 18 trials, 8 were control trials on which the numbers presented during the probe display were completely unique from those presented on the prime display. Another 8 trials were ignored repetition trials on which the target on the probe display matched the distractor on the prime display. Finally, the remaining 2 trials were number 9 (or letter $P$ ) trials in which the prime display contained the number 9 (or the letter $\mathrm{P}$ ) as a distractor stimulus.

Apparatus and Stimuli. An IBM-486-compatible microcomputer with a 14-in. color VGA monitor was used for all testing. The program was written in MEL 2.0, and response times (RTs) were measured as the interval between the stimulus onset and the participant's manual response. Both RTs and error rates were recorded.

The stimuli consisted of the numbers $1-9$ and the letter $P$, all measuring approximately $8 \mathrm{~mm}$ high $\times 5 \mathrm{~mm}$ wide and presented in a stroked New York font. These stimuli were viewed from a distance of approximately $80 \mathrm{~cm}$.

We created ignored-repetition trials by first assigning stimuli to roles as if a control trial were being generated, then changing the distractor of the prime display to match the target on the probe display. This ensured that keypress changes to target items would be roughly equated across trials, without any systematic bias across the experiments as a whole.

\section{Results}

Prior to performing any inferential statistics, a check for outliers was conducted on the basis of the overall mean RTs. As a result, data were removed for 3 participants in the monitor-distractor condition of Experiment 1B, whose RTs fell more than 2.5 standard deviations $(S D \mathrm{~s})$ above the group mean. This left 12 participants per condition per experiment. Table 1 presents the resulting means and $S D$ s for both RT and error rate.

The participants in the monitor-distractor conditions of Experiments 1A and 1B responded correctly on 79\% and $87 \%$ of the number 9 and letter P trials, respectively. These proportions were significantly different $[t(22)=$ $2.62, p<.05]$, suggesting that it was slightly easier for the participants to notice the presence of the stimulus that was semantically unique from those in the primary task (i.e., a letter presented in the context of a number task). In addition, the relatively high performance in both task contexts provides strong support that the participants were indeed monitoring the distractors as instructed.

Response time analyses. To examine the effects of monitoring on negative priming, we performed a $2 \times 2 \times 2$ ANOVA that focused on the effects of trial type (control vs. ignored repetition), condition (ignore-distractor vs. monitordistractor), and the identity of the to-be-monitored-for item 
Table 1

Mean Response Times (RTs, in Milliseconds) and Percent Errors Across Conditions of Experiments $1 \mathrm{~A}$ and $1 \mathrm{~B}$

\begin{tabular}{|c|c|c|c|c|c|c|c|c|}
\hline & \multicolumn{4}{|c|}{ Ignore Distractor } & \multicolumn{4}{|c|}{ Monitor Distractor } \\
\hline & RT & $S D$ & $\%$ Error & $S D$ & RT & $S D$ & $\%$ Error & $S D$ \\
\hline \multicolumn{9}{|l|}{ Monitor for 9} \\
\hline Number 9 & 652 & 138 & 1.8 & 2.4 & 1,047 & 228 & 1.3 & 2.6 \\
\hline Ignored repetition & 685 & 139 & 3.0 & 2.1 & 945 & 162 & 2.8 & 2.3 \\
\hline Control & 671 & 141 & 2.7 & 1.7 & 901 & 152 & 2.7 & 2.1 \\
\hline Negative priming & 14 & & 0.3 & & 44 & & 0.1 & \\
\hline \multicolumn{9}{|l|}{ Monitor for $\mathrm{P}$} \\
\hline Letter P & 690 & 108 & 5.0 & 3.7 & 1,059 & 397 & 1.3 & 2.1 \\
\hline Ignored repetition & 719 & 134 & 4.3 & 1.9 & 934 & 288 & 3.6 & 2.4 \\
\hline Control & 696 & 120 & 4.3 & 2.5 & 906 & 307 & 3.3 & 2.3 \\
\hline Negative priming & 23 & & 0 & & 28 & & 0.3 & \\
\hline \multicolumn{9}{|l|}{ Collapsed } \\
\hline Number 9/P & 671 & 123 & 3.4 & 3.5 & 1,053 & 317 & 1.3 & 2.3 \\
\hline Ignored repetition & 702 & 135 & 3.7 & 2.0 & 939 & 229 & 3.2 & 2.3 \\
\hline Control & 683 & 129 & 3.5 & 2.2 & 903 & 237 & 3.0 & 2.2 \\
\hline Negative priming & 19 & & 0.2 & & 36 & & 0.2 & \\
\hline
\end{tabular}

(P vs. 9). This analysis was performed only on those trials on which responses were accurate to both the prime and the probe targets and did not include trials in which the prime contained the to-be-monitored-for item.

The analysis revealed significant main effects for both condition $\left[F(1,44)=17.08, M S_{\mathrm{e}}=73,486, p<.001\right]$ and trial type $\left[F(1,44)=15.36, M S_{\mathrm{e}}=1,168, p<.001\right]$. Responses were slower in the monitor-distractor condition than in the ignore-distractor condition and were slower on ignored repetition trials than on control trials (i.e., significant negative priming). The interaction between condition and trial type was not significant $[F(1,44)=1.62]$, suggesting equal magnitudes of negative priming across the two conditions.

Error rate analyses. These data were analyzed via the same $2 \times 2 \times 2$ mixed design ANOVA performed on the RTs. This analysis revealed only a slight trend toward a main effect of the identity of the monitoring stimulus $\left[F(1,44)=3.6, M S_{\mathrm{e}}=0.0008, p<.07\right]$, with performance being slightly more accurate in the context of the number 9 stimulus than in the context of the letter P stimulus $(M S=$ $97.6 \%$ and $96.4 \%$, respectively). However, this factor did not interact with any of the other factors (all $F_{\mathrm{s}}<2.8$ ), nor were any of the other main effects or interactions significant. Thus, the error rate was stable across conditions.

\section{Discussion}

Of primary importance, we again observed negative priming when the participants were encouraged to attend to and process distractors. While the negative priming observed was numerically larger in the monitor-distractor condition, this augmentation of the effect was not statistically reliable. Finally, although the participants were slightly better at spotting the Ps relative to the $9 \mathrm{~s}$ when they occurred as to-be-monitored-for distractor stimuli, this distinction did not affect the negative priming in any statistically reliable manner. In general, the first claim of MacDonald et al. (1999) - that attending to distractors does not eliminate negative priming — was supported.
With respect to the second claim of MacDonald et al. (1999), there was no direct evidence in the present study of the negative priming effect being augmented by encouraging participants to attend to the distractors. That is, there was no interaction between trial type and condition. That said, to the extent that attending to the distractor had any effect, it was to increase the size of the negative priming from a borderline effect in the ignore-distractor condition to a robustly significant one in the monitor-distractor condition, at least in terms of RT. We do not wish to overemphasize this, but it does agree with the suggestion of MacDonald et al. that if attending to the distractors affects negative priming at all, it acts to strengthen the effect.

\section{GENERAL DISCUSSION}

The purpose of the present work was to revisit the conclusions made by MacDonald et al. (1999). Specifically, those conclusions consisted of two central claims surrounding the effects of directing attention toward distractors in the context of otherwise typical negative priming procedures. First, MacDonald et al. claimed that negative priming persisted despite distractors being attended, suggesting that it was not the attentional status of the critical item per se that gave rise to negative priming. Second, MacDonald et al. found that the negative priming effect became much larger when attention was directed to the distractors, suggesting that attention to items that were not responded to might actually cause negative priming effects to become larger.

Given the work of Mackintosh et al. (2002) suggesting that the specific referent-size task used by MacDonald et al. (1999) may support artifacts, we reexamined the two claims highlighted in the previous paragraph using a different procedure. The results support the first claim, in that negative priming clearly persisted even when attention was directed toward distractors. With respect to the second claim, however, we did not observe a reliable augmentation of the negative priming effect when atten- 
tion was directed toward distractors. Although a slight numerical increase in the effect was observed, this increase was not statistically reliable and was nowhere near the magnitude of increase observed by MacDonald et al. This suggests that much of the augmentation MacDonald et al. observed might indeed have been related to the specific task used.

Note that these findings are not necessarily bad news for any current account of negative priming. Many accounts, such as Neill and Mathis's (1998) transfer-inappropriate processing account, the selection-feature mismatch account of MacDonald and Joordens (2000), and Milliken, Joordens, Merikle, and Seiffert's (1998) temporaldiscrimination theory rely on the distractors being encoded during the prime display, and attention should enhance such encoding. Thus, these theories are quite consistent with the notion that attention could enhance or be necessary for negative priming.

As discussed in the introduction, even the selectionfor-action accounts of negative priming (Tipper et al., 1999; Tipper et al., 1994) do not demand the assumption that critical items are selected against in a traditional selective-attention sense. The competitive processes that Tipper and colleagues highlight could occur later in the processing stream and depend on the previous distractor item having been reasonably encoded. Thus, these processes are also consistent with the finding that attending to the distractors does not eliminate negative priming.

The point of this study was not to discriminate between theories but rather it was to clarify what role attention plays in determining negative priming effects. Our primary conclusion is that negative priming does not result from distractors being unattended since it is observed even when procedures are used that encourage participants to attend to distractors. Thus, it appears that the critical aspect of typical negative priming procedures is the categorizing of stimuli into those that are to be responded to versus those that are not.

Although initial theories of selective attention posited that selection occurred very early in stimulus processing with "unattended" stimuli receiving little processing at all (e.g., Broadbent, 1958), later studies suggested that stimuli may be processed deeply prior to any selection occurring. In fact, theories that assume a very late locus of selection often do tie selective attention into response goals and mechanisms (e.g., Deutsch \& Deutsch, 1963). Therefore, one might argue that a late-selection view of selective attention is synonymous with selection for action, and, in fact, early negative priming studies did frame the negative priming phenomenon in terms of a lateselection viewpoint (e.g., Neill, 1977; Tipper, 1985).

However, we counter that it is not appropriate to equate a late-selection view of attention with the notion of selection for action because doing so removes the relevance of distinguishing between attending to, versus not attending to, a stimulus. In a number of studies, it has been shown that items processed under focused versus divided attention give rise to data patterns that parallel those that occur when participants perceive the same stimuli with versus without awareness (Geng, Zhu, \& Li, 2001; Klinger, 2001; Merikle \& Joordens, 1997). These studies provide empirical support for the notion that attention mediates awareness. When one adds to this the notion that a stimulus that has been attended might or might not be responded to, then it appears clear to us that processes of response selection are at least partially distinct from those underlying selective attention.

Hence, our primary point is the following. If mechanisms of selective attention are even partly distinct from mechanisms of selective responding, then it is imperative that researchers be clear about which of these mechanisms a particular phenomenon may reflect. With this in mind, the present findings show that the negative priming phenomenon does not reflect a selective-attention mechanism because attended items give rise to at least as much negative priming as do unattended items. Thus, researchers of negative priming should be careful to restrict their interpretations to mechanisms of selective responding.

\section{REFERENCES}

Allport, A. (1987). Selection for action: Some behavioral and neurophysiological considerations of attention and action. In H. Heuer \& A. F. Sanders (Eds.), Perspectives on perception and action (pp. 395419). Hillsdale, NJ: Erlbaum.

Broadbent, D. E. (1958). Perception and communication. London: Pergamon.

Broadbent, D. E. (1965). Information theory and perception. Anthropology \& Medicine, 13, 369-379.

Cherry, C. E. (1953). Some experiments on the recognition of speech, with one and with two ears. Journal of the Acoustical Society of America, 25, 975-979.

Cherry, C. E., \& TAylor, W. K. (1954). Some further experiments on the recognition of speech with one and with two ears. Journal of the Acoustical Society of America, 26, 554-559.

DAlRymple-Alford, E. C., \& BudAyr, B. (1966). Examination of some aspects of the Stroop color-word test. Perceptual \& Motor Skills, 23, 1211-1214.

Deutsch, J. A., \& Deutsch, D. (1963). Attention: Some theoretical considerations. Psychological Review, 70, 80-90.

Geng, H., ZHU, Y., \& LI, Y. (2001). False recognition: Awareness, attention and stimulus quality. Acta Psychologica Sinica, 33, 104-110.

KLINGER, M. R. (2001). The roles of attention and awareness in the false recognition effect. American Journal of Psychology, 114, 93-114.

MacDonald, P. A., \& JoORdens, S. (2000). Investigating a memorybased account of negative priming: Support for selection feature mismatch. Journal of Experimental Psychology: Human Perception \& Performance, 26, 1478-1496.

MacDonald, P. A., Joordens, S., \& Seergobin, K. N. (1999). Negative priming effects that are bigger than a breadbox: Attention to distractors does not eliminate negative priming, it enhances it. Memory \& Cognition, 27, 197-207.

Mackintosh, B., Mathews, A., \& Holden, E. (2002). Bigger than a breadbox? Attention to distractors may not enhance negative priming. Journal of Experimental Psychology: Human Perception \& Performance, 28, 1323-1329.

Merikle, P. M., \& Joordens, S. (1997). Parallels between perception without attention and perception without awareness. Consciousness \& Cognition, 6, 219-236.

Milliken, B., \& Joordens, S. (1996). Negative priming without overt prime selection. Canadian Journal of Experimental Psychology, 50, 333-346.

Milliken, B, Joordens, S., Merikle, P. M., \& Seiffert, A. E. (1998). Selective attention: A reevaluation of the implications of negative priming. Psychological Review, 105, 203-229.

NEILL, W. T. (1977). Inhibition and facilitation processes in selective 
attention. Journal of Experimental Psychology: Human Perception \& Performance, 3, 444-450.

NeiLl, W. T., \& Mathis, K. M. (1998). Transfer-inappropriate processing: Negative priming and related phenomena. In D. L. Medin (Ed.), The psychology of learning and motivation: Advances in research and theory (Vol. 38, pp. 1-44). San Diego: Academic Press.

TIPPER, S. P. (1985). The negative priming effect: Inhibitory effects of ignored primes. Quarterly Journal of Experimental Psychology, 37A, 571-590.

Tipper, S. P., Howard, L. A., \& Houghton, G. (1999). Action-based mechanisms of attention. In G. W. Humphreys, J. Duncan, \& A. Treisman (Eds.), Attention, space, and action: Studies in cognitive neuroscience (pp. 232-247). Oxford: Oxford University Press.

Tipper, S. P., Weaver, B., \& Houghton, G. (1994). Behavioural goals determine inhibitory mechanisms of selective attention. Quarterly Journal of Experimental Psychology, 47A, 809-840

Treisman, A. (1964). Selection attention in man. British Medical Journal, 20, 12-16.

Treisman, A. (1996). Selection for perception for selection for action. Visual Cognition, 3, 353-357.

Treisman, A., Squire, R., \& Green, J. (1974). Semantic processing in dichotic listening? A replication. Memory \& Cognition, 2, 641-646.

van der Heisden, A. H. C. (1996). Perception for selection, selection for action, and action for perception. Visual Cognition, 3, 357-361.

(Manuscript received February 25, 2005;

revision accepted for publication August 11, 2005.) 